\title{
Eigenkapitalkostensatz mit dem CAPM berechnen
}

\author{
Vor allem große börsennotierte Unternehmen nutzen das Capital Asset Pricing Model (CAPM), \\ um im Rahmen der finanziellen Unternehmensführung den Eigenkapitalkostensatz zu bestim- \\ men. Nicht börsennotierte Unternehmen wenden es weniger oft an, da es für sie schwieriger \\ ist, ihr Risiko mit dem Kapitalmarktmodell zu ermitteln. Ein modifizierter Berechnungsansatz \\ zeigt exemplarisch am Beispiel von Aldi Süd, wie sie das kapitalmarktbezogene Verfahren des \\ CAPMs dennoch für sich nutzbar machen können.
}

\section{Enzo Mondello}

Das Capital Asset Pricing Model (CAPM) kann in der finanziellen Unternehmensführung im Rahmen der Investitionsanalyse eingesetzt werden. Mit dem Kapitalmarktmodell lässt sich der Eigenkapitalkostensatz beziehungsweise der Kapitalkostensatz ermitteln. Übersteigt die Rendite des Investitionsvorhabens den projektspezifischen Kapitalkostensatz, wird ein Mehrwert für das Unternehmen geschaffen. Darüber hinaus

\section{Zusammenfassung}

- Die Berechnung des Eigenkapitalkostensatzes mit dem CAPM eignet sich auch für nicht börsennotierte Unternehmen, wenn man das Bottom-up-Beta nutzt.

- Im Vergleich zum historischen Beta ist das Bottomup-Beta stabiler, da es aus einem Durchschnittswert hervorgeht.

- Das Bottom-up-Beta erfasst das aktuelle Risiko eines Unternehmens zudem besser, weil Veränderungen des Geschäftsrisikos und des finanziellen Risikos in der Risikogröße enthalten sind. kann der Kapitalkostensatz angewendet werden, um die optimale Kapitalstruktur festzulegen und die Bewertung von Unternehmen vorzunehmen. Obwohl sich das aus den 1960er Jahren stammende CAPM auf vereinfachende Annahmen stützt, ist das Modell in der Wirtschaftspraxis weitverbreitet: Über 70 Prozent der börsennotierten multinationalen Unternehmen in Europa nutzen das CAPM für die Berechnung des Eigenkapitalkostensatzes (vergleiche Bancel/Mittoo 2004, S. 103 ff.). Kleinere Unternehmen, die nicht an einer Börse notiert sind, wenden das CAPM weniger häufig an (vergleiche Brounen/de Jong/ Koedijk 2004, S. 71 ff.). Das ist nicht überraschend, weil es für nicht börsennotierte Unternehmen, also solche, deren Aktien nicht an der Börse gehandelt werden, schwieriger ist, das systematische Risiko beziehungsweise das Beta zu ermitteln. Da hier eine Regression historischer Renditedaten nicht möglich ist, wird ein Beta mithilfe der Betas börsennotierter Vergleichsunternehmen bestimmt. So können auch nicht börsennotierte Unternehmen ein kapitalmarktbezogenes Verfahren zur Schätzung des Eigenkapitalkostensatzes verwenden.

Gemäß dem Kapitalmarktmodell hängt die erwartete Rendite der Eigenkapitalgeber beziehungsweise der Eigenkapital- 
kostensatz lediglich vom systematischen Risiko ab, das dem Marktrisiko entspricht und mit dem Beta des Beteiligungspapiers gemessen wird. Unsystematische Risiken spielen keine Rolle, da das CAPM auf der Annahme beruht, dass die Investoren gut diversifiziert sind, sodass sie lediglich dem Marktrisiko ausgesetzt sind. Je größer das Marktrisiko beziehungsweise das Beta ist, desto höher fällt die erwartete Rendite aus.

Abbildung 1 veranschaulicht die Berechnungsweise des Eigenkapitalkostensatzes mit dem CAPM. Damit der Eigenkapitalkostensatz berechnet werden kann, ist zum langfristigen risikolosen Zinssatz die Risikoprämie hinzuzuzählen, die aus dem Produkt der Marktrisikoprämie mit dem Beta besteht.

In den folgenden Ausführungen wird gezeigt, wie der Eigenkapitalkostensatz mit dem CAPM sowohl für das börsennotierte Unternehmen Daimler als auch für das nicht börsennotierte Unternehmen Aldi Süd ermittelt werden kann. Die Berechnungen erfolgen zu Beginn des Jahres 2019. Dabei wird für den langfristigen risikolosen Zinssatz die Verfallrendite 30-jähriger deutscher Bundesanleihen von 0,75 Prozent verwendet. Die erwartete Marktrisikoprämie von 6,25 Prozent richtet sich nach den Empfehlungen des Fachausschusses für Unternehmensbewertung und Betriebswirtschaft (FAUB), der zu Beginn des Jahres 2019 eine Bandbreite für die Marktrisikoprämie von 5,5 bis sieben Prozent (Mittelwert von 6,25 Prozent) angegeben hat. In der Zwischenzeit hat der FAUB am 25. Oktober 2019 aufgrund der andauernden expansiven Geldpolitik der Europäischen Zentralbank (EZB) und den damit zusammenhängenden negativen Zinsen seine bisherige Empfehlung für die Marktrisiko- prämie auf sechs bis acht Prozent angehoben (vergleiche Institut für Wirtschaftsprüfer 2019). Die wirtschaftlichen negativen Auswirkungen der COVID-19-Pandemie sind gemäß dem FAUB eher kurzfristiger Natur und spielen daher bei langfristig orientierten Bewertungsmodellen eine untergeordnete Rolle.

\section{CAPM-Eigenkapitalkostensatz für Daimler}

Für das börsennotierte Unternehmen Daimler lässt sich der CAPM-Eigenkapitalkostensatz sowohl mit dem historischen Beta als auch mit dem Bottom-up-Beta ermitteln. Die Bestimmung des historischen Betas erfolgt über eine einfache lineare Regressionsanalyse. Für die Regression werden im vorliegenden Beispiel 60 monatliche stetige Renditen der Automobil-Aktie und des DAX 30 verwendet. Die monatlichen Renditen beziehen sich auf eine fünfjährige Zeitperiode der monatlichen Kursdaten von Ende März 2014 bis Ende März 2019. Aus der Regression resultiert folgende Regressionsgleichung für die Daimler-Aktie:

$\mathrm{r}_{\text {Daimler, } \mathrm{t}}=-0,924 \%+1,506 \mathrm{r}_{\mathrm{DAX}, \mathrm{t}}+€_{\text {Daimler, }} \cdot$

Abbildung 2 (S. 58) zeigt die Regressionsgerade mit der entsprechenden Regressionsstatistik. Der Determinationskoeffizient der Regression beträgt 0,736, was auf einen hohen Erklärungsgehalt der Regression hinweist. Die Steigung der Regressionsgeraden von 1,506 entspricht dem Beta der Aktie. Diese misst die Veränderung der Aktienrendite hinsichtlich einer Veränderung der Marktrendite. Die t-Statistik für die Steigung

\section{Abb. 1 Eigenkapitalkostensatz mit dem CAPM berechnen}

Eigenkapitalkostensatz $=$

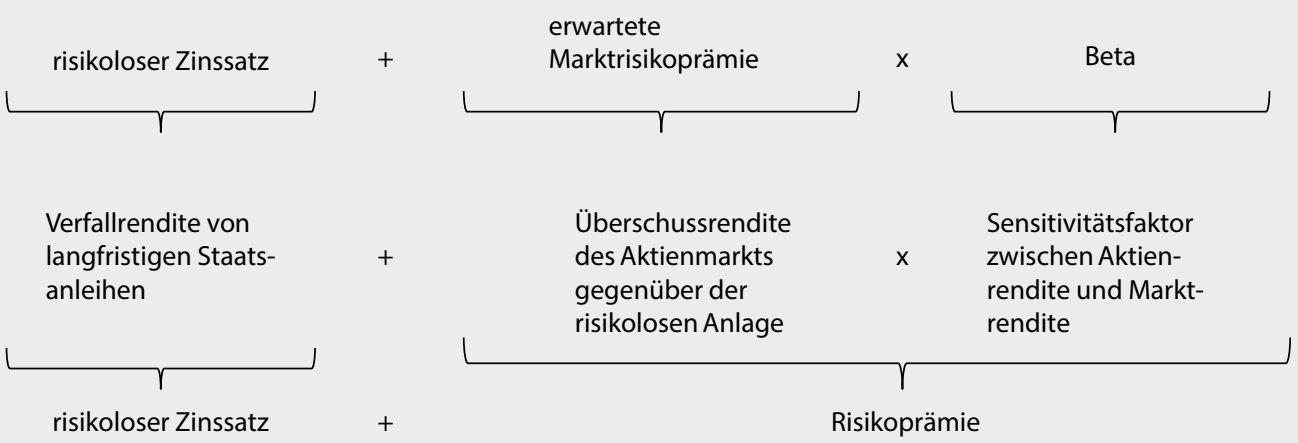

Quelle: eigene Darstellung 
der Regressionsgerade beläuft sich auf 12,724. Die t-Statistik liegt bei einem Signifikanzniveau von fünf Prozent über dem kritischen $\mathrm{t}$-Wert von rund 2. Demnach ist das Beta statistisch signifikant.

Empirisch betrachtet folgen die Betakoeffizienten von Aktien über die Zeit allerdings keiner Zufallsbewegung, sondern bewegen sich im Durchschnitt gegen ihren Erwartungswert von 1. Daher wird das historische Beta mit folgender Formel gegen 1 korrigiert, was für die Daimler-Aktie ein adjustiertes Beta von 1,338 ergibt (vergleiche Blume 1971, S. 8 f.):

$\beta_{\text {adjustiert }}=0,333+0,667 \times 1,506=1,338$.

Der Eigenkapitalkostensatz von 9,11 Prozent lässt sich mithilfe des CAPMs wie folgt bestimmen:

$\mathrm{K}_{\mathrm{EK}, \text { Daimler }}=0,75 \%+6,25 \% \times 1,338=9,11 \%$.

Der CAPM-Eigenkapitalkostensatz lässt sich auch mit dem Bottom-up-Beta berechnen (vergleiche Damodaran 2015, S. $122 \mathrm{ff}$.). Damit das Bottom-up-Beta bestimmt werden kann, sind in einem ersten Schritt die vergleichbaren börsennotierten Unternehmen auszuwählen, die im gleichen Geschäftsbereich tätig sind (vergleiche Tabelle 1).

In einem zweiten Schritt wird das Asset Beta berechnet, eine um die finanzielle Verlustgefahr bereinigte Risikogröße, die das systematische Geschäftsrisiko der Industrie beziehungsweise der Benchmark-Gesellschaften wiedergibt. Für die Bestimmung des Asset Betas werden der Median für das Beta von 1,25, für den Verschuldungsgrad von 134,4 Prozent und für den Grenzertragssteuersatz von 30 Prozent genommen, was ein Asset Beta von 0,644 ergibt (vergleiche Hamada 1972, S. 435 ff.):

$\beta_{\text {Asset }}=1,25 /[1+(1-0,30) \times 1,344]=0,644$.

In einem dritten Schritt wird das Asset Beta der Vergleichsunternehmen von den flüssigen Mitteln bereinigt, da diese mehrheitlich Liquiditätsreserven umfassen und demnach für die betriebliche Tätigkeit nicht erforderlich sind (vergleiche Damodaran 2015, S. 122):

$\beta_{\text {Asset Betrieb }}=0,644 /(1-0,183)=0,788$.

Da davon ausgegangen wird, dass die flüssigen Mittel ein Beta von null besitzen und somit über kein Marktrisiko verfügen, ist das Asset Beta der betrieblichen Vermögenswerte von 0,788 höher als das Asset Beta der gesamten Vermögenswerte von 0,644 . Des Weiteren wird unterstellt, dass alle Vergleichsunternehmen über ein ähnliches operatives Risiko verfügen, das dem Median des Verhältnisses zwischen den Fixkosten und variablen Kosten von 22,5 Prozent entspricht. Daher wird in einem vierten Schritt das um das operative Ri-

Abb. 2 Regression zwischen den monatlichen Renditen der Daimler-Aktie und dem DAX

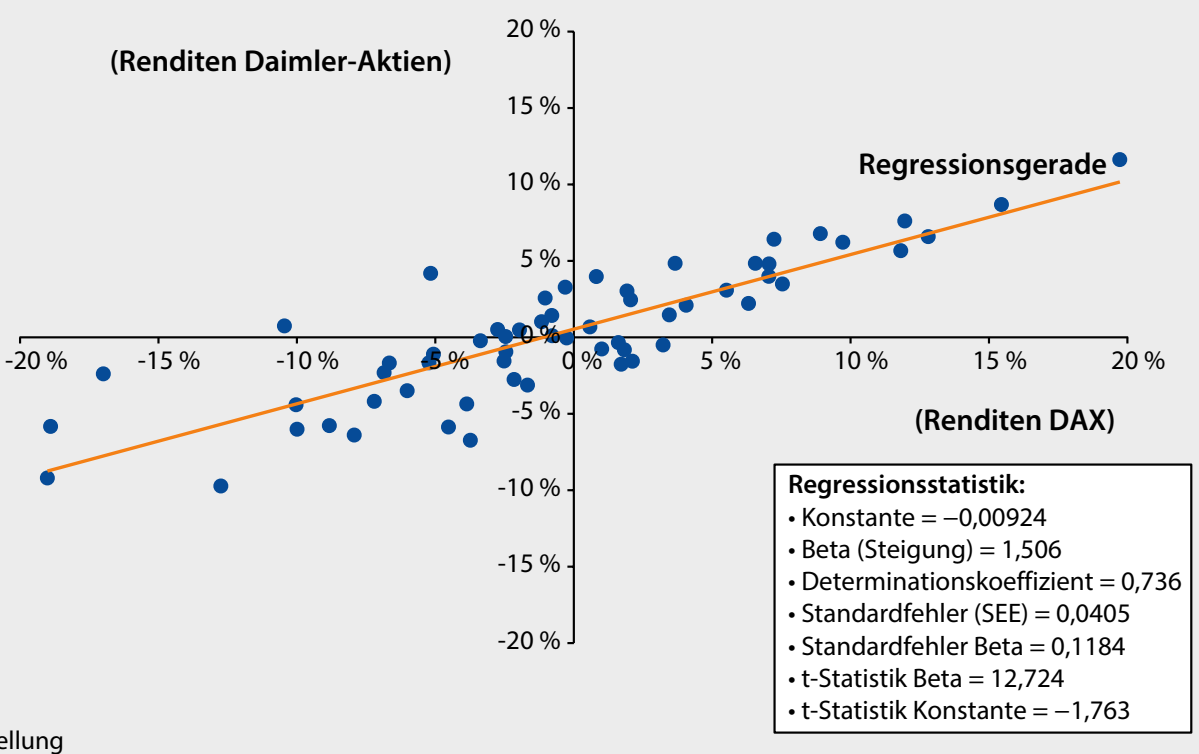

Quelle: eigene Darstellung 
siko korrigierte Asset Beta der betrieblichen Vermögenswerte wie folgt berechnet (vergleiche Damodaran 2012, S. 199):

$\beta_{\text {Asset Betrieb korrigiert }}=0,788 / 1,225=0,643$.

In Schritt fünf wird das um das finanzielle und operative Risiko sowie das um den nicht operativen Teil der Vermögenswerte korrigierte Asset Beta der Vergleichsgesellschaften von 0,643 mit dem operativen Risiko beziehungsweise dem Verhältnis zwischen den Fixkosten und variablen Kosten von Daimler adjustiert:

$\beta_{\text {Asset Betrieb, Daimler }}=0,643 \times 1,175=0,756$.

Schließlich wird das Bottom-up-Beta von Daimler bestimmt, indem das Asset Beta der betrieblichen Vermögenswerte mit dem Grenzertragssteuersatz und dem angestrebten Verhält- nis zwischen Fremd- und Eigenkapital des Unternehmens wie folgt angepasst wird:

$\beta_{\text {Bottom-up, Daimler }}=0,756 \times[1+(1-0,3) \times 2,546]=2,10$.

Das Bottom-up-Beta umfasst neben dem systematischen beziehungsweise dem nicht diversifizierbaren Geschäftsrisiko, das für alle Unternehmen in der Industrie im Durchschnitt gleich hoch ist, auch das systematische finanzielle Risiko des Automobilbauers. Der Eigenkapitalkostensatz für Daimler von 13,88 Prozent kann mit dem CAPM folgendermaßen ermittelt werden:

$\mathrm{K}_{\mathrm{EK}, \text { Daimler }}=0,75 \%+6,25 \% \times 2,10=13,88 \%$.

Das Bottom-up-Beta besitzt im Vergleich zum historischen Beta einige Vorteile (vergleiche Mondello 2017, S. 99):

\section{Tab. 1 Daimler und dessen 14 wichtigste globale börsennotierte Vergleichsunternehmen}

\begin{tabular}{|c|c|c|c|c|c|}
\hline Unternehmen & $\begin{array}{c}\text { historisches } \\
\text { Beta }\end{array}$ & $\begin{array}{l}\text { Verschuldungsgrad: } \\
\text { Verhältnis Fremd- zu } \\
\text { Eigenkapital }\end{array}$ & $\begin{array}{l}\text { Grenzertrags- } \\
\text { steuersatz }\end{array}$ & $\begin{array}{l}\text { flüssige Mittel in \% } \\
\text { des Unternehmens- } \\
\text { werts }\end{array}$ & $\begin{array}{l}\text { operatives Risiko: } \\
\text { Verhältnis Fixkosten zu } \\
\text { variablen Kosten }\end{array}$ \\
\hline Daimler & 1,51 & $254,6 \%$ & $30 \%$ & $13,2 \%$ & $17,5 \%$ \\
\hline BMW & 1,28 & $209,7 \%$ & $30 \%$ & $11,4 \%$ & $29,2 \%$ \\
\hline Volkswagen & 1,46 & $229,8 \%$ & $30 \%$ & $21,2 \%$ & $37,6 \%$ \\
\hline Renault & 1,51 & $66,9 \%$ & $33 \%$ & $22,3 \%$ & $18,1 \%$ \\
\hline Peugeot & 1,67 & $37,8 \%$ & $33 \%$ & $36,4 \%$ & $15,0 \%$ \\
\hline Fiat Chrysler & 1,43 & $69,1 \%$ & $19 \%$ & $26,9 \%$ & $19,9 \%$ \\
\hline Toyota & 1,09 & $103,4 \%$ & $34 \%$ & $12,1 \%$ & $27,9 \%$ \\
\hline General Motors & 1,31 & $197,4 \%$ & $27 \%$ & $16,2 \%$ & $27,0 \%$ \\
\hline Tesla & 0,34 & $27,1 \%$ & $27 \%$ & $6,4 \%$ & $39,6 \%$ \\
\hline SAIC Motors & 0,63 & $63,3 \%$ & $25 \%$ & $25,9 \%$ & $18,4 \%$ \\
\hline Honda Motor & 1,24 & $134,4 \%$ & $34 \%$ & $18,3 \%$ & $26,5 \%$ \\
\hline Nissan Motor & 1,22 & $186,0 \%$ & $34 \%$ & $10,8 \%$ & $22,0 \%$ \\
\hline Hyundai Motor & 1,25 & $183,9 \%$ & $25 \%$ & $23,5 \%$ & $18,9 \%$ \\
\hline Ford Motor & 1,04 & $252,2 \%$ & $27 \%$ & $17,6 \%$ & $22,5 \%$ \\
\hline Kia Motors & 1,15 & $30,6 \%$ & $25 \%$ & $35,6 \%$ & $24,3 \%$ \\
\hline einfacher Mittelwert & 1,21 & $136,4 \%$ & $29 \%$ & $19,9 \%$ & $24,3 \%$ \\
\hline Median & 1,25 & $134,4 \%$ & $30 \%$ & $18,3 \%$ & $22,5 \%$ \\
\hline
\end{tabular}


- Das Bottom-up-Beta lässt sich auch für nicht börsennotierte Unternehmen ermitteln, bei denen keine historische Renditezeitreihe vorliegt. So kann es vor einem ersten Börsengang und für Geschäftsbereiche eines Unternehmens geschätzt werden.

- Verändert sich die Zusammensetzung der Geschäftsbereiche, kann man dies in die Bottom-up-Beta-Berechnungen einfließen lassen, indem ein Asset Beta mit der Summe der gewichteten Asset Betas der einzelnen Geschäftsbereiche bestimmt wird. Sieht die strategische Stoßrichtung des Unternehmens neue Geschäftsbereiche in der Zukunft vor, können diese zukünftigen Entwicklungen in die Ermittlung des Betas einbezogen werden.

- Das historische Beta umfasst das durchschnittliche Verhältnis zwischen Fremd- und Eigenkapital während der Regressionsperiode. Ändert sich die Kapitalstruktur, wird diese Veränderung erst mit der Zeit, über neuere Renditedaten, in das Beta eingebunden. Das Bottom-up-Beta hingegen stützt sich auf den aktuellen oder erwarteten Verschuldungsgrad.

- Das historische Beta einzelner Aktien ist nicht stabil und weist einen großen Schätzfehler auf (vergleiche Levy 1971, S. 55 ff.). Beim Bottom-up-Beta wird ein Durchschnittswert von mehreren historischen Betas gebildet, das zu einem stabileren Beta mit geringerem Schätzfehler führt.

\section{CAPM-Eigenkapitalkostensatz für Aldi Süd}

Für Daimler ist eine Korrektur des Betas um das unsystematische Risiko nicht erforderlich, weil die Aktionärsstruktur des Automobilunternehmens mehrheitlich aus institutionellen Investoren mit einem Anteil von 52,3 Prozent besteht, die gut di- versifiziert sind. Private Investoren halten lediglich einen Anteil von 23,1 Prozent an Daimler (vergleiche Daimler 2020). Im Gegensatz dazu ist Aldi Süd im Besitz einer Familienstiftung (Siepmann-Stiftung), die wenig diversifiziert ist. Um das Bottom-up-Beta zu bestimmen, sind analog zu Daimler die vergleichbaren börsennotierten Unternehmen auszuwählen. Die europäischen Vergleichsunternehmen stammen aus dem Sektor Lebensmitteleinzelhandel (vergleiche Tabelle 2).

Für Aldi Süd wird ein Verschuldungsgrad auf Basis von Marktwerten von 30 Prozent unterstellt. Das Verhältnis von Fixkosten zu variablen Kosten beläuft sich auf 21 Prozent (Quelle: Bundesanzeiger und eigene Berechnungen). Der Grenzertragssteuersatz liegt bei 30 Prozent.

Das Asset Beta von 0,740 lässt sich mithilfe des Medians für das Beta von 0,93, für den Verschuldungsgrad von 33 Prozent und für den Grenzertragssteuersatz von 22 Prozent wie folgt berechnen:

$\beta_{\text {Asset }}=0,93 /[1+(1-0,22) \times 0,33]=0,740$.

Das um den Median des Verhältnisses zwischen dem Wert der flüssigen Mittel und dem Unternehmenswert bereinigte Asset Beta liegt bei 0,855:

$\beta_{\text {Asset Betrieb }}=0,740 /(1-0,135)=0,855$.

Wird das Asset Beta der betrieblichen Vermögenswerte um das operative Risiko korrigiert, gelangt man zu einem Wert von 0,673 :

\section{Tab. 2 Börsennotiere Vergleichsunternehmen aus dem Sektor Lebensmitteleinzelhandel}

\begin{tabular}{|c|c|c|c|c|c|c|}
\hline Unternehmen & $\begin{array}{l}\text { histori- } \\
\text { sches Beta }\end{array}$ & $\begin{array}{c}\text { Determi- } \\
\text { nations- } \\
\text { koeffizient }\end{array}$ & $\begin{array}{l}\text { Verschuldungsgrad: } \\
\text { Verhältnis Fremd- } \\
\text { zu Eigenkapital }\end{array}$ & $\begin{array}{c}\text { Grenzertrags- } \\
\text { steuersatz }\end{array}$ & $\begin{array}{l}\text { flüssige Mittel } \\
\text { in \% des Unter- } \\
\text { nehmenswerts }\end{array}$ & $\begin{array}{l}\text { operatives Risiko: } \\
\text { Verhältnis Fix- zu } \\
\text { variablen Kosten }\end{array}$ \\
\hline J Sainsbury & 0,90 & 0,14 & $36 \%$ & $19 \%$ & $27,93 \%$ & $28,32 \%$ \\
\hline Wm Morrison Supermarkets & 0,67 & 0,10 & $25 \%$ & $19 \%$ & $4,05 \%$ & $24,18 \%$ \\
\hline Tesco & 1,01 & 0,16 & $30 \%$ & $19 \%$ & $10,64 \%$ & $25,86 \%$ \\
\hline Koninklijke Ahold Delhaize & 0,73 & 0,19 & $27 \%$ & $25 \%$ & $11,70 \%$ & $37,16 \%$ \\
\hline Carrefour & 0,96 & 0,36 & $102 \%$ & $33 \%$ & $15,30 \%$ & $24,89 \%$ \\
\hline Casino Guichard Perrachon & 1,13 & 0,30 & $229 \%$ & $33 \%$ & $20,52 \%$ & $28,70 \%$ \\
\hline einfacher Mittelwert & 0,90 & 0,21 & $75 \%$ & $25 \%$ & $15,02 \%$ & $28,19 \%$ \\
\hline Median & 0,93 & 0,175 & $33 \%$ & $22 \%$ & $13,50 \%$ & $27,09 \%$ \\
\hline
\end{tabular}


$\beta_{\text {Asset Betrieb korrigiert }}=0,855 / 1,2709=0,673$.

Das um das operative Risiko korrigierte Asset Beta der betrieblichen Vermögenswerte von 0,673 wird mit dem operativen Risiko beziehungsweise dem Verhältnis zwischen den Fixkosten und variablen Kosten von Aldi Süd angepasst:

$\beta_{\text {Asset Betrieb, Aldi Süd }}=0,673 \times 1,21=0,814$.

Mit dem Einbezug des finanziellen Risikos resultiert ein Bottom-up-Beta für Aldi Süd von 0,985:

$\beta_{\text {Bottom-up, Aldi Süd }}=0,814 \times[1+(1-0,3) \times 0,3]=0,985$.

Das Bottom-up-Beta erfasst lediglich denjenigen Anteil am Gesamtrisiko, der sich nicht diversifizieren lässt. Für den Lebensmitteleinzelhandel erklärt das systematische Risiko nur einen kleinen Teil der Renditevarianz $\left(\mathrm{R}^{2}=0,175\right)$. Da die Eigentümer von Aldi Süd (Familienstiftung) wenig diversifiziert sind, ist das Bottom-up-Beta um das im Vergleich zum systematischen Risiko höhere Gesamtrisiko zu korrigieren (vergleiche Damodaran 2015, S. 136):

$\beta_{\text {Gesamtrisiko, Aldi Süd }}=0,985 / \sqrt{ } 0,175=2,355$.

Für das nicht börsennotierte Unternehmen Aldi Süd ergibt sich anhand der vorliegenden Berechnungen ein Eigenkapitalkostensatz mit dem CAPM von 15,47 Prozent:

$\mathrm{K}_{\mathrm{EK}, \text { Aldi Süd }}=0,75 \%+6,25 \% \times 2,355=15,47 \%$.

Das höhere Beta für das Gesamtrisiko (im Beispiel von Aldi Süd von 2,355 gegenüber 0,985) führt zu einem höheren und somit realistischeren Eigenkapitalkostensatz für ein Unternehmen, dessen Eigentümer wenig diversifiziert sind.

\section{Fazit}

Mit dem CAPM kann nicht nur der Eigenkapitalkostensatz für börsennotierte, sondern auch für nicht börsennotierte Unternehmen ermittelt werden. Hierzu ist ein Bottom-up-Beta zu bestimmen, das aus den historischen Betas von Vergleichsunternehmen festgelegt wird. Im Vergleich zum historischen Beta, das nur für börsennotierte Unternehmen berechnet werden kann, ist das Bottom-up-Beta stabiler, da es aus einem Durchschnittswert hervorgeht. Darüber hinaus umfasst es das aktuelle Risiko des Unternehmens besser als das historische
Beta, weil Veränderungen des Geschäftsrisikos und des finanziellen Risikos in der Risikogröße enthalten sind. Im Gegensatz dazu werden Risikoveränderungen im historischen Beta erst mit der Zeit beziehungsweise mit neuen Renditedaten in der Regression berücksichtigt. Daher stellt das Bottom-upBeta die geeignetere Risikogröße für die Berechnung des Eigenkapitalkostensatzes mit dem CAPM dar. Darüber hinaus kann das Beta korrigiert werden, wenn das Unternehmen im Besitz von nicht diversifizierten Eigentümern ist.

\section{Literatur}

Bancel, F./Mittoo, U. R. (2004): Cross-country determinants of capital structure choice: a survey of european firms, in: Financial Management, 33 (4), S. 103-132.

Blume, M. E. (1971): On the assessment of risk, in: Journal of Finance, 26 (1), S. 1-10.

Brounen, D./de Jong, A./Koedijk, K. C. (2004): Corporate finance in Europe: confronting theory with practice, in: Financial Management, 33 (4), S. 71-101.

Daimler (2020): Daimler-Aktionäre im Überblick, www.daimler. com/investoren/aktie/aktionaersstruktur (letzter Abruf: 15.05.2020).

Damodaran, A. (2015): Applied corporate finance, 4. Auflage, Hoboken.

Damodaran, A. (2012): Investment valuation: tools and techniques for determining the value of any asset, 3. Auflage, Hoboken.

Hamada, R. S. (1972): The effect of the firm's capital structure on the systematic risk of common stocks, in: Journal of Finance, 27 (2), S.435-452.

Institut der Wirtschaftsprüfer (2019): Neue Kapitalkostenempfehlungen des FAUB, https://tinyurl.com/kapitalkostenempfehlungenFAUB (letzter Abruf 15.05.2020).

Levy, R. A. (1971): On the short-term stationarity of beta coefficients, in: Financial Analysts Journal, 27 (6), 55-62.

Mondello, E. (2017): Aktienbewertung: Theorie und Anwendungsbeispiele, 2. Auflage, Wiesbaden.

www.springerprofessional.de/link/13324842

\section{Angaben zum Autor}

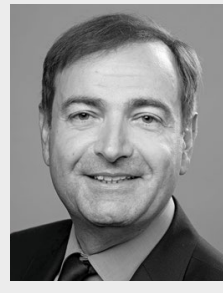

Dr. Enzo Mondello

ist Inhaber und Managing Director von CfBS Center for Business Studies AG sowie Lehrbeauftragter an der Universität St. Gallen und Dozent an der Kalaidos Fachhochschule Schweiz.

E-Mail:mondello@cf-studies.ch 